\title{
A eficácia da solução de EDTA na remoção de smear layer e sua relação com o tempo de uso: uma revisão integrativa
}

The effectiveness of EDTA solution in removing smear layer and its
relation to the time of use: an integrative review

\author{
Shelly Coura Mafra* \\ Caroline Felipe M. Girelli** \\ Viviane Ferreira G. Xavier ${ }^{* * *}$ \\ Mariane Floriano Lopes Lacerda*** \\ Gabriel Pinheiro Lacerda ${ }^{* * * *}$ \\ Renato Girelli Coelho ${ }^{* * * * *}$
}

\section{Resumo}

Objetivo: o presente trabalho teve como objetivo identificar qual o tempo de aplicação mais frequentemente utilizado do EDTA para remoção eficaz da smear layer no interior dos canais radiculares e quais os fatores que podem influenciar na ação do EDTA. Metodologia: foi realizada uma revisão integrativa de literatura, utilizando-se as bases de dados Scielo, Lilacs, PubMed e Science Direct com os seguintes descritores: EDTA, irrigação final (final rinse), camada de esfregaço (smear layer), tempo de irrigação (time of irrigation), descacificação (decalcification) e microdureza (microhardness). Os critérios de inclusão foram artigos publicados na íntegra, no idioma Inglês e Português, durante o período de janeiro de 2000 a janeiro de 2017. Resultados: após análise baseada nos critérios de inclusão, leitura dos resumos disponíveis e avaliação sobre o questionamento elaborado, foram identificadas no Scielo um total de 507 referências bibliográficas, as quais, três foram selecionadas. Já no Lilacs foram identificados 35 artigos, sendo considerados dois. No PubMed foram identificadas 450 referências, sendo considerada apenas uma. Foram encontrados 282 artigos na Science Direct, sendo selecionados 24. Desta forma, 30 artigos fizeram parte da presente revisão. Conclusão: com base na literatura estudada, verificou-se que e o tempo de uso do
EDTA interferiu diretamente na qualidade da remoção da smear layer. Além disso, verificou-se que o EDTA a $17 \%$ utilizado por um período de um minuto foi ideal para a remoção da smear layer, enquanto que a aplicação de EDTA no tempo superior a sete minutos provocou erosão da dentina peritubular.

Palavras-chave: EDTA. Camada de Esfregaço. Quelantes.

\section{Introdução}

O sucesso do tratamento endodôntico está intimamente relacionado à qualidade da limpeza e desinfecção do sistema de canais radiculares (SCR), obtido por meio da eliminação ou pelo menos redução do conteúdo orgânico e inorgânico contaminado do interior dos canais ${ }^{1,2}$. Para atingir tais objetivos, os canais são submetidos ao preparo químico mecânico (PQM).

O PQM é considerado um requisito chave para o sucesso da terapia endodôntica, uma vez que consiste na limpeza e modelagem -clean and shape- do

Especialista em Saúde Coletiva, Especialista em Endodontia da ABO-GV (Associação brasileira de Odontologia)

Mestre em endodontia, Coordenadora e Professora da especialização em endodontia da ABO-GV (Associação Brasileira de Odontologia). Especialista em Endodontia, Professora da UNIVALE (Universidade Vale do Rio Doce).

Doutora em Endodontia, professora departamento de Odontologia UFJF (Universidade Federal de Juiz de Fora), Governador Valadares, MG.

Mestre em Prótese, do Departamento de Odontologia UFJF (Universidade Federal de Juiz de Fora) Governador Valadares.

Doutor em Ciências Odontológicas, Professor da UNIVALE (Universidade Vale do Rio Doce), Governador Valadares. 
canal radicular e suas eventuais ramificações, através da instrumentação mecânica, complementada pelas substâncias irrigadoras e medicação intracanal; removendo a maior quantidade possível de detritos, restos pulpares, micro-organismos; criando condições ideais para que ocorra a recuperação e regeneração tecidual ${ }^{3}$.

Pesquisas ao longo dos anos têm demonstrado que a instrumentação, seja ela rotatória ou manual, tem a capacidade de gerar um magma residual, tanto orgânico como inorgânico no interior dos canais, denominado de smear layer ${ }^{4}$. A smear layer contem vestígios de raspas de dentina, tecido pulpar e prolongamentos odontoblásticos e, no caso de dentes infectados ou contaminados por saliva, de bactérias; é constituída por uma camada superficial, fracamente aderida à parede radicular, que apresenta espessura média de 1 a $2 \mu \mathrm{m}$, enquanto que a outra, mais profunda, está intimamente ligada aos túbulos dentinários, obliterando-os, podendo chegar a $40 \mu^{4}$.

A presença dessa camada residual na superfície dentinária pode gerar efeitos deletérios ao tratamento, pois retarda a ação de medicamentos intracanais e de substâncias químicas auxiliares no interior de túbulos dentinários; ação essa, de suma importância, visto que nem todas as paredes do canal sofrem ação dos instrumentos, permanecendo áreas completamente intocadas e micro-organismos não atingidos pelo $\mathrm{PQM}$ irão reinfectar os canais, culminando no insucesso da terapia endodôntica ${ }^{4}$. Além disso, embora tenha sido sugerido que uma intacta smear layer pode impedir a penetração inicial de bactérias nos túbulos dentinários, a sua degradação após o tratamento contribui para essa reinfecção. Para tanto, faz-se necessário, a remoção desta smear layer, não só do SCR, mas também, das porções peritubular e intertubular ${ }^{5,6}$.

Com o propósito de remover a smear layer, diversas soluções irrigadoras vêm sendo estudadas. $\mathrm{O}$ hipoclorito de sódio $(\mathrm{NaOCl})$ tem sido a solução mais utilizada para dissolução do componente orgânico do magma residual ${ }^{6}$. Contudo, por não agir sobre a parte inorgânica, torna-se necessário complementar o seu uso com uma solução que possua ação quelante $\mathrm{e}^{7,8}$.

Introduzido no final dos anos 50, o ácido etilenodiaminotetraacético, comumente conhecido por EDTA, é o quelante mais utilizado na Odontologia. Esta solução, que tem eficiência comprovada na dissolução de material inorgânico, ${ }^{9}$ age por quelação, sequestrando íons Cálcio da dentina, formando quelatos de cálcio solúveis, promovendo assim, uma descalcificação de 20 a $30 \mu \mathrm{m}$ de profundidade ${ }^{10-16}$.

Apesar de ser amplamente utilizado, não há um consenso sobre o tempo para que o EDTA faça a descalcificação e remoção da smear layer de maneira eficaz, podendo variar, de acordo com os protocolos estipulados, de um a 15 minutos sobre o tempo de aplicação do EDTA $^{15}$.
Com a finalidade de sintetizar os resultados obtidos em pesquisas sobre esse tema em questão, de maneira sistemática, ordenada e abrangente, a revisão integrativa de literatura fornece informações mais amplas sobre o assunto/problema, proporcionando como resultado, um quadro completo de conceitos complexos ${ }^{16}$. Diante disto, este trabalho teve por objetivo identificar, por meio de uma revisão integrativa, qual o tempo de aplicação mais frequentemente utilizado do EDTA para remoção eficaz da smear layer no interior dos canais radiculares, bem como os fatores que irão influenciar na ação do EDTA.

\section{Métodos}

Optou-se pela revisão integrativa da literatura, definida como um método por meio dos quais os resultados de pesquisas são sumarizados, o que possibilita síntese, categorização, análise e avaliação do conhecimento científico já produzido sobre o tema ${ }^{17}$. Tal síntese, viabiliza a contextualização do pesquisador sobre a temática, além de sinalizar lacunas do conhecimento que precisam ser preenchidas com novos estudos, contribuindo assim, para discussões sobre métodos e resultados de pesquisas anteriores ${ }^{18}$.

Para a elaboração da revisão integrativa, no primeiro momento, o revisor determina o objetivo, formula os questionamentos a serem respondidos e, então, realiza a busca para identificar e coletar o máximo de pesquisas primárias relevantes dentro dos critérios de inclusão e exclusão previamente estabelecidos ${ }^{19}$.

Desta forma, para nortear a realização deste estudo, formulou-se a seguinte questão: qual o tempo mais frequentemente utilizado do EDTA para remoção eficaz da smear layer no interior dos canais radiculares?

Para o levantamento bibliográfico deste estudo, fez-se uma busca online nas bases de dados Scielo (Biblioteca Eletrônica de Periódicos Científicos Brasileiros), Lilacs (Literatura Latino-Americana em Ciências da Saúde), PubMed (base de dados desenvolvida pelo National Center for Biotechnology Information na National Library of Medicine) e Science Direct, utilizando os seguintes descritores: EDTA, irrigação final (final rinse), camada de esfregaço (smear layer), tempo de irrigação (time of irrigation), descacificação (descalcification) e microdureza (microhardness). Vale salientar que "magma dentinário, lama dentinária e detritos", foram utilizados como sinônimo do "material sedimentado após o corte da dentina radicular".

Também foi executada a busca manual por quatro revisores, após a leitura do título, resumo e palavras-chave. Quando as informações contidas nesses tópicos foram insuficientes, os artigos foram lidos na íntegra. As referências dos estudos, possíveis 
de serem utilizados, bem como a busca cruzada da base de dados dos autores, serviram de guia para a seleção de novos artigos relevantes.

Como critério de inclusão, tanto eletrônico quanto manual, foram selecionados artigos de revisão de literatura ou sistemática e de pesquisa in vivo, ou in vitro, publicados na íntegra, entre os anos 2000 a 2017 e nos idiomas inglês e português. Para verificar se os artigos atendiam a esses critérios, realizou-se uma avaliação por dois revisores independentes, autores deste artigo, sendo as avaliações subsequentemente comparadas. Nesse momento, também foram identificados e excluídos os estudos que apresentavam duplicidade entre as bases.

Após leitura dos artigos selecionados, todos os registros foram armazenados em um arquivo específico do programa Microsoft Excel 2007 e as informações foram coletadas e digitadas em banco de dados de acordo com as seguintes variáveis: artigo, autores/ano, tempo de uso do EDTA, concentração do EDTA, volume de EDTA, forma de irrigação, presença ou ausência de agitação do EDTA, análise da smear layer residual e conclusão.

\section{Resultados}

Foram identificadas no Scielo um total de 507 referências bibliográficas, sendo selecionados três artigos. Já no Lilacs foram identificadas 35 referências e considerados dois artigos. No PubMed, foram identificadas 450 referências, permanecendo 19 artigos. Foram encontrados 282 artigos na Science Direct, sendo selecionados 24. Desta forma, 30 artigos fizeram parte da presente revisão (Quadro 1).

Quanto ao delineamento do estudo, $100 \%$ dos artigos foram do tipo transversal e $100 \%$ estruturados na língua inglesa. Em relação ao ano de pu- blicação, 13,3\% foram publicados em 2017; 23,3\% em 2016; $30 \%$ em 2015; 13,3\% em 2014 e os demais (20\%) publicados entre os anos de 2002 a 2010. A maioria foi publicada nos EUA $(66,8 \%)$ e os demais na Índia (20\%), China (3,3\%), Canadá (3,3\%), Suíça $(3,3 \%)$ e Grã-Bretanha (3,3\%).

No que tange a metodologia, 26 dos 30 artigos selecionados utilizaram o EDTA na concentração de $17 \%$. A concentração de $5 \%$ foi utilizada em um único caso $^{20}$, assim como a concentração de $15 \%{ }^{21}$ e as concentrações 10 e $20 \%{ }^{22,23}$. Em todos os artigos, o EDTA foi conduzido por meio de irrigação com seringa, sendo que em um único estudo, o EDTA entrou em contato com a superfície do dente, por meio de imersão.

Ao analisar em qual sequência as soluções foram empregadas, verificou-se que o EDTA foi utilizado como irrigação final seguido do uso de $\mathrm{NaOCl}$ na maioria dos estudos $(73,3 \%)$. Nos demais casos, o soro fisiológico ${ }^{24}$ e a água destilada ${ }^{20,23,25-29}$ prosseguiram a irrigação após o uso do quelante.

Em relação à associação do EDTA com outras substâncias, registrou-se o uso de $\mathrm{MTAD}^{24}$ (composto por uma mistura de doxicilina, ácido cítrico e um detergente Tween-80), EDTA-T³ (EDTA + detergente Tergentol), EDTA-C ${ }^{25}$ (EDTA+ tensoativo Cetavlon) e Smear Clear ${ }^{30}$ (EDTA + dois tensoativos).

Dois outros critérios, volume e tempo, também foram levados em consideração, durante a análise dos estudos selecionados. Diferentes volumes foram identificados para a irrigação final de EDTA: $1 \mathrm{~mL}$, 2,5 mL, $3 \mathrm{~mL}, 5 \mathrm{~mL}, 6 \mathrm{~mL}, 10 \mathrm{~mL}$ e $20 \mathrm{~mL}$. No entanto, a maior diversidade foi encontrada em relação ao tempo de uso do EDTA, o qual variou de 15 segundos até 15 minutos.

Para avaliar a quantidade de smear layer residual após a utilização do EDTA, em 100\% dos estudos, a microscopia eletrônica de varredura (MEV) foi o método escolhido.

Quadro 1 - Identificação dos estudos selecionados para a revisão integrativa

\begin{tabular}{|c|c|c|c|c|c|c|c|c|}
\hline Artigo & Autor/ano & Tempo & Concentração & Volume & $\begin{array}{l}\text { Forma de } \\
\text { irrigação }\end{array}$ & $\begin{array}{l}\text { Ativação } \\
\text { do edta }\end{array}$ & Análise & Conclusão \\
\hline $\begin{array}{l}\text { Time- dependent effects of } \\
\text { EDTA on dentin structures }\end{array}$ & Çalt ${ }^{54}, 2002$ & $\begin{array}{l}1,10 \mathrm{mi}- \\
\text { nutos }\end{array}$ & EDTA a $17 \%$ & $10 \mathrm{ML}$ & SERINGA & NÃO & MEV & $\begin{array}{l}\text { EDTA a } 17 \% \text { por } 1 \text { minuto foi } \\
\text { eficaz na remoção de smear } \\
\text { layer e } 10 \text { minutos causou } \\
\text { erosão na camada peritubular. }\end{array}$ \\
\hline $\begin{array}{l}\text { Effect of three different time } \\
\text { periods of irrigation with } \\
\text { EDTA-T, EDTA, and citric } \\
\text { acid on smear layer removal }\end{array}$ & $\begin{array}{l}\text { Scelza et al. }{ }^{3}, \\
2004\end{array}$ & $\begin{array}{l}3,10,15 \\
\text { minutos }\end{array}$ & EDTA a $17 \%$ & $20 \mathrm{ML}$ & SERINGA & NÃO & MEV & $\begin{array}{l}\text { Os } 3 \text { irrigantes foram eficazes } \\
\text { no menor espaço de tempo } \\
\text { testado e não demonstrou efei- } \\
\text { to melhorado com o aumento } \\
\text { do tempo. }\end{array}$ \\
\hline $\begin{array}{l}\text { The effect of application time } \\
\text { of EDTA and NaOCl on intra- } \\
\text { canal Smear Layer removal: } \\
\text { an SEM analysis }\end{array}$ & $\begin{array}{l}\text { Teixeira et } \\
\text { al. }^{21}, 2005\end{array}$ & $\begin{array}{l}1,3,5 \\
\text { minutos }\end{array}$ & EDTA a $15 \%$ & $3 \mathrm{ML}$ & SERINGA & NÃO & MEV & $\begin{array}{l}\text { EDTA por } 1,3 \text { e } 5 \text { minutos } \\
\text { foram igualmente eficazes na } \\
\text { remoção da smear layer. }\end{array}$ \\
\hline $\begin{array}{l}\text { Effect of EDTA with and } \\
\text { without surfactants or ultra- } \\
\text { sonics on removal of Smear } \\
\text { Layer }\end{array}$ & $\begin{array}{l}\text { Lui et al. }{ }^{30} \text {, } \\
2007\end{array}$ & 1 minuto & EDTA a $17 \%$ & $5 \mathrm{ML}$ & SERINGA & Ultrassom & MEV & $\begin{array}{l}\text { Adição de surfactantes ao EDTA } \\
\text { em Smear Clear não resultou em } \\
\text { melhor remoção da smear layer. } \\
\text { Uso de ultrassons com EDTA a } \\
17 \% \text { aumentou a remoção. }\end{array}$ \\
\hline $\begin{array}{l}\text { A comparative study of sme- } \\
\text { ar layer removal and erosion } \\
\text { in apical intraradicular den- } \\
\text { tine with three irrigating so- } \\
\text { lutions: a scanning electron } \\
\text { microscopy evaluation }\end{array}$ & $\begin{array}{l}\text { Mancini et } \\
\text { al. }^{50}, 2009\end{array}$ & 1 minuto & EDTA a $17 \%$ & $1 \mathrm{ML}$ & SERINGA & NÃO & MEV & $\begin{array}{l}\text { Um minuto não foi suficiente } \\
\text { para remover a camada de } \\
\text { esfregaço, para nenhuma das } \\
\text { substâncias, especialmente no } \\
\text { terço apical e erosão não foi } \\
\text { encontrada }\end{array}$ \\
\hline
\end{tabular}




\begin{tabular}{|c|c|c|c|c|c|c|c|c|}
\hline $\begin{array}{l}\text { Influence of final rinse tech- } \\
\text { nique on ability of ethylene- } \\
\text { diaminetetraacetic acid of } \\
\text { removing smear layer }\end{array}$ & $\begin{array}{l}\text { Mello et al. }{ }^{24} \text {, } \\
2010\end{array}$ & 3 minutos & EDTA a $17 \%$ & $5 \mathrm{ML}$ & SERINGA & NÃO & MEV & $\begin{array}{l}\text { Irrigação contínua com } 5 \mathrm{ml} \\
\text { de EDTA a } 17 \% \text { por } 3 \mathrm{mi}- \\
\text { nutos foi mais eficiente para } \\
\text { remover a smear layer das } \\
\text { paredes dos canais. }\end{array}$ \\
\hline $\begin{array}{l}\text { Smear layer removal efficacy } \\
\text { of different irrigating solu- } \\
\text { tions: A comparative scan- } \\
\text { ning electron microscope } \\
\text { evaluation }\end{array}$ & $\begin{array}{l}\text { Ahir et al. }{ }^{75}, \\
2014\end{array}$ & 1 minuto & EDTA a $17 \%$ & $1 \mathrm{ML}$ & SERINGA & NÃO & MEV & $\begin{array}{l}\text { Os quelantes avaliados foram } \\
\text { eficazes em remover a smear } \\
\text { layer do canal, no entanto } \\
\text { nenhum deles foi capaz de } \\
\text { deixar o terço apical livre do } \\
\text { esfregaço. }\end{array}$ \\
\hline $\begin{array}{l}\text { Effectiveness of Various Ir- } \\
\text { rigation Activation Protocols } \\
\text { and the Self-Adjusting File } \\
\text { System on Smear Layer and } \\
\text { Debris Removal }\end{array}$ & $\begin{array}{l}\text { Çapar et al. }{ }^{64}, \\
2014\end{array}$ & 1 minuto & EDTA a $17 \%$ & $10 \mathrm{ML}$ & $\begin{array}{l}\text { SERINGA/ } \\
\text { SERINGA } \\
+ \text { NAVYTIP }\end{array}$ & \begin{tabular}{l|} 
Endoactivator \\
(EA) \\
Endovac (EV) \\
Self-adjusting \\
file (SAF) \\
PUI
\end{tabular} & MEV & $\begin{array}{l}\text { Agitação da soluções com as } \\
\text { técnicas EA, EV, SAF e PUI } \\
\text { produziu paredes mais limpas } \\
\text { em comparação com airriga- } \\
\text { ção convencionale sistemas } \\
\text { Navytip. }\end{array}$ \\
\hline $\begin{array}{l}\text { An in-vitro evaluation of } \\
\text { effect of EDTAC on root den- } \\
\text { tin with respect to time. }\end{array}$ & $\begin{array}{l}\text { Darda et al. }{ }^{25} \\
2014\end{array}$ & $\begin{array}{l}1,10 \\
\text { minutos }\end{array}$ & $\begin{array}{l}\text { EDTAC a } \\
17 \%\end{array}$ & $10 \mathrm{ML}$ & SERINGA & NÃO & MEV & $\begin{array}{l}\text { EDTA-C a } 17 \% \text { por } 1 \text { minuto } \\
\text { é eficaz para a remoção de } \\
\text { smear layer, enquanto que por } \\
10 \text { minutos provoca a erosão } \\
\text { da dentina peritubular }\end{array}$ \\
\hline $\begin{array}{l}\text { Efficacy of solution form of } \\
\text { ethylenediaminetetraace- } \\
\text { tic acid on removing smear } \\
\text { layer of root canal at different } \\
\text { exposure time in vitro }\end{array}$ & $\begin{array}{l}\text { Poudyal et } \\
\text { al. }{ }^{51}, 2014\end{array}$ & $\begin{array}{l}1,3,5,7 \\
\text { minutos }\end{array}$ & EDTA a $17 \%$ & $5 \mathrm{ML}$ & SERINGA & NÃO & MEV & $\begin{array}{l}\text { EDTA a } 17 \%+\mathrm{NaOCl} \text { a } 2,5 \% \\
\text { durante } 7 \text { minutos removeu } \\
\text { quase completamente a sme- } \\
\text { ar layer. } 3 \text { e } 5 \text { minutos hou- } \\
\text { ve remoção parcial e em } 1 \\
\text { minuto percebe-se remoção } \\
\text { negligenciável }\end{array}$ \\
\hline $\begin{array}{l}\text { The effect of the temperature } \\
\text { changes of EDTA and MTAD } \\
\text { on the removal of the smear } \\
\text { layer: a scanning llectron mi- } \\
\text { croscopy study }\end{array}$ & $\begin{array}{l}\text { Çiçek et al. }{ }^{76} \\
2015\end{array}$ & - & EDTA a $17 \%$ & $5 \mathrm{ML}$ & SERINGA & NÃO & MEV & $\begin{array}{l}\text { Usando EDTA e MTAD a } 25^{\circ} \mathrm{C} \\
\text { e } 37^{\circ} \mathrm{C} \text { encontrou maior eficá- } \\
\text { cia do que a temperatura de } \\
4^{\circ} \mathrm{C} \text {, mesmo no nível apical } \\
\text { para remoção da smear layer. }\end{array}$ \\
\hline $\begin{array}{l}\text { Comparison of different irri- } \\
\text { gation activation techniques } \\
\text { on smear layer removal: an } \\
\text { in vitro study }\end{array}$ & $\begin{array}{l}\text { Ekin et al. }{ }^{27}, \\
2015\end{array}$ & 80 segundos & EDTA a $17 \%$ & $6 \mathrm{ML}$ & SERINGA & $\begin{array}{l}\text { LASER, PIPS } \\
\text { e PUI }\end{array}$ & MEV & $\begin{array}{l}\text { Ativação com PUI, Nd: YAG, } \\
\text { Er: YAG e PIPS demonstrou- } \\
\text {-se eficaz na remoção de } \\
\text { smear layer }\end{array}$ \\
\hline $\begin{array}{l}\text { Influence of Diode Laser Ap- } \\
\text { plication on the Efficiency of } \\
\text { QMiX and EDTA Solutions in } \\
\text { Removing Smear Layer }\end{array}$ & $\begin{array}{l}\text { Koçak et al. }{ }^{56}, \\
2015\end{array}$ & 1 minuto & EDTA a $17 \%$ & $5 \mathrm{ML}$ & SERINGA & LASER & MEV & $\begin{array}{l}\text { Nenhuma solução removeu } \\
\text { completamente a smear layer, } \\
\text { especialmente nos terços api- } \\
\text { cais. Laser diodo diminuiu a } \\
\text { quantidade de smear layer }\end{array}$ \\
\hline $\begin{array}{l}\text { Comparison of efficacy of } \\
\text { various root canal irrigation } \\
\text { systems in removal of sme- } \\
\text { ar layer generated at apical } \\
\text { third: An SEM study }\end{array}$ & $\begin{array}{l}\text { Kumar et al. }{ }^{33}, \\
2015\end{array}$ & 1 minuto & EDTA a $17 \%$ & - & SERINGA & $\begin{array}{l}\text { Endovac, } \\
\text { Endoactivator } \\
\text { e max I Probe }\end{array}$ & MEV & $\begin{array}{l}\text { EndoVac e EndoActivator exe- } \\
\text { cutaram muito melhor do que } \\
\text { outros sistemas disponíveis na } \\
\text { remoção da camada de esfre- } \\
\text { gaço do terceiro apical. Então } \\
\text { eles devem ser incorporados } \\
\text { como uma parte regular do re- } \\
\text { gime de irrigação. }\end{array}$ \\
\hline $\begin{array}{l}\text { A comparative evaluation } \\
\text { of smear layer removal by } \\
\text { using edta, etidronic acid, } \\
\text { and maleic acid as root canal } \\
\text { irrigants: An in vitro scanning } \\
\text { electron microscopic study }\end{array}$ & $\begin{array}{l}\text { Kuruvilla et } \\
\text { al. }^{29}, 2015\end{array}$ & 1 minuto & EDTA a $17 \%$ & $5 \mathrm{ML}$ & SERINGA & NÃO & MEV & $\begin{array}{l}\text { Todos os irrigantes removeram } \\
\text { a camada de esfregaço dos di- } \\
\text { ferentes níveis (coronal, médio } \\
\text { e apical). Ácido maleico a } 7 \% \\
\text { foi mais eficiente que EDTA a } \\
17 \% \text { e ácido etidrónico a } 18 \% \\
\text { na remoção de smear layer } \\
\text { do1/3 apical }\end{array}$ \\
\hline $\begin{array}{l}\text { Comparison of Smear Layer } \\
\text { Removal Using the Nd:YAG } \\
\text { Laser, Ultrasound, ProTa- } \\
\text { per Universal System, and } \\
\text { CanalBrush Methods: An In } \\
\text { Vitro Study }\end{array}$ & $\begin{array}{l}\text { Lima et al. }{ }^{65}, \\
2015\end{array}$ & 30 segundos & EDTA a $17 \%$ & $1 \mathrm{ML}$ & SERINGA & $\begin{array}{l}\text { Agitação } \\
\text { com lima, } \\
\text { canal brush, } \\
\text { LASER, PUI, }\end{array}$ & MEV & $\begin{array}{l}\text { Nenhum dos métodos de agi- } \\
\text { tação fez completa remoção } \\
\text { de smear layer. O ultrassom } \\
\text { melhorou significativamente } \\
\text { quando comparado ao laser } \\
\text { Nd: YAG, o Canal Brush e } \\
\text { lima, no } 1 / 3 \text { apical }\end{array}$ \\
\hline $\begin{array}{l}\text { Comparison of positive-pres- } \\
\text { sure, passive ultrasonic, and } \\
\text { laser-activated irrigations on } \\
\text { smear-layer removal from the } \\
\text { root canal surface }\end{array}$ & $\begin{array}{l}\text { Sahar-Helfit et } \\
\text { al. }^{66}, 2015\end{array}$ & 1 minuto & EDTA a $17 \%$ & $10 \mathrm{ML}$ & SERINGA & LASER e PUI & MEV & $\begin{array}{l}\text { Ativação com laser Er: YAG } \\
\text { foim o étodo mais eficiente } \\
\text { para remover a camada de } \\
\text { esfregaço de toda a parede } \\
\text { do canal radicular. }\end{array}$ \\
\hline $\begin{array}{l}\text { Effect of Ultrasonic Activa- } \\
\text { tion of Irrigants on Smear } \\
\text { Layer Removal }\end{array}$ & $\begin{array}{l}\text { Schmidt et al., } \\
2015\end{array}$ & 3 minutos & EDTA a $17 \%$ & $3 \mathrm{ML}$ & SERINGA & PUI & MEV & $\begin{array}{l}\text { A utilizaçãoo da PUI aumentou } \\
\text { a remoção de smear layer quan- } \\
\text { do utilizado com o EDTA. Ao } \\
\text { ser utilizado com o } \mathrm{NaOCI} \text { não } \\
\text { apresentou diferença estatística } \\
\text { com e sem a PUI }\end{array}$ \\
\hline
\end{tabular}




\begin{tabular}{|c|c|c|c|c|c|c|c|c|}
\hline $\begin{array}{l}\text { Evaluation of the smear layer } \\
\text { removal and erosive capacity } \\
\text { of EDTA, boric acid, citric } \\
\text { acid and desy clean solu- } \\
\text { tions: an in vitro study }\end{array}$ & $\begin{array}{l}\text { Turk et al. }{ }^{20}, \\
2015\end{array}$ & 1 minuto & EDTA a $5 \%$ & $5 \mathrm{ML}$ & SERINGA & NÃO & MEV & $\begin{array}{l}\text { Desy Clean pode ser um agente } \\
\text { promissor como solução de ir- } \\
\text { rigação com capacidade de re- } \\
\text { moção da smear layer e menos } \\
\text { efeitos erosivos. }\end{array}$ \\
\hline $\begin{array}{l}\text { Effect of diode laser and ultra- } \\
\text { sonics with and without ethy- } \\
\text { lenediaminetetraacetic acid } \\
\text { on smear layer removal from } \\
\text { the root canals: A scanning } \\
\text { electron microscope study }\end{array}$ & $\begin{array}{l}\text { Amin et al. }{ }^{67} \text {, } \\
2016\end{array}$ & $\begin{array}{l}20 \text { segundos } \\
\text { LASER/ } 1 \\
\text { minuto } \\
\text { ultrassom }\end{array}$ & EDTA a $17 \%$ & $1 \mathrm{ML}$ & SERINGA & $\begin{array}{l}\text { LASER e } \\
\text { Ultrassom }\end{array}$ & MEV & $\begin{array}{l}\text { Todos os grupos testados fo- } \\
\text { ram capazes de remover smear } \\
\text { layer exceto } \mathrm{NaOCl} \text { +ultras- } \\
\text { som. Laser de diodo sozinho, } \\
\text { foi melhor que o ultrassom. } \\
\text { O laser diodo pode ser usado } \\
\text { para emoversmear layer }\end{array}$ \\
\hline $\begin{array}{l}\text { Comparative analysis of en- } \\
\text { dodontic smear layer removal } \\
\text { efficacy of } 17 \% \text { ethylenedia- } \\
\text { minetetraacetic acid, } 7 \% \text { ma- } \\
\text { leic acid, and } 2 \% \text { chlorhexi- } \\
\text { dine using scanning electron } \\
\text { microscope: An in vitro study }\end{array}$ & $\begin{array}{l}\text { Attur et al. }{ }^{26}, \\
2016\end{array}$ & 1 minuto & EDTA a $17 \%$ & $5 \mathrm{ML}$ & SERINGA & NÃO & MEV & $\begin{array}{l}\text { O presente estudo mostrou } \\
\text { que EDTA a } 17 \% \text { removeu } \\
\text { eficientemente a camada de } \\
\text { esfregaço das paredes do ca- } \\
\text { nal radicular. }\end{array}$ \\
\hline $\begin{array}{l}\text { Effectiveness of laser-assisted } \\
\text { irrigation and Passive Ultra- } \\
\text { sonic Irrigation techniques } \\
\text { on Smear Layer removal in } \\
\text { middle and apical thirds }\end{array}$ & $\begin{array}{l}\text { Ayranci et } \\
\text { al. }^{68}, 2016\end{array}$ & 1 minuto & EDTA a $17 \%$ & $2,5 \mathrm{ML}$ & SERINGA & $\begin{array}{l}\text { LASER e } \\
\text { ultrassom }\end{array}$ & MEV & $\begin{array}{l}\text { LASER com a combinação } \\
\text { de } \mathrm{NaOCl} \text { a } 2,5 \% \text { e EDTA } \\
\text { a } 17 \% \text { removeu melhor a } \\
\text { smear layer que a PUI com a } \\
\text { mesma combinação }\end{array}$ \\
\hline $\begin{array}{l}\text { Effect of time and ultrasonic } \\
\text { activation on ethylenediami- } \\
\text { netetraacetic acid on smear } \\
\text { layer removal of the root } \\
\text { canal }\end{array}$ & $\begin{array}{l}\text { Castro et al. }{ }^{63} \text {, } \\
2016\end{array}$ & $\begin{array}{l}20 \text { segundos } \\
\text { e } 60 \text { segun- } \\
\text { dos }\end{array}$ & EDTA a $17 \%$ & $2,5 \mathrm{ML}$ & SERINGA & PUI & MEV & $\begin{array}{l}\text { A associação EDTA } 17 \% \text { e Na- } \\
\text { OCl 2,5\% foi mais importante } \\
\text { do que o tempo de exposição } \\
\text { ao EDTA. Irrigação convencio- } \\
\text { nal e PUI têm eficácia igual }\end{array}$ \\
\hline $\begin{array}{l}\text { The effectiveness of the } \\
\text { Erbium: Yttrium aluminum } \\
\text { garnet PIPStechnique in com- } \\
\text { parison to different chemi- } \\
\text { cal solutionsin removing the } \\
\text { endodontic smear layer-an in } \\
\text { vitroprofilometric study }\end{array}$ & $\begin{array}{l}\text { Nasher et } \\
\text { al. }^{22}, 2016\end{array}$ & 30 segundos & EDTA a $20 \%$ & $3 \mathrm{ML}$ & SERINGA & PIPS & MEV & $\begin{array}{l}\text { Não houve diferença estatísti- } \\
\text { ca entre os grupos com e sem } \\
\text { ativação da solução na remo- } \\
\text { ção de smear layer }\end{array}$ \\
\hline $\begin{array}{l}\text { To Evaluate the Efficacy of } \\
\text { an Innovative Irrigant on } \\
\text { Smear Layer Removal - SEM } \\
\text { Analysis }\end{array}$ & $\begin{array}{l}\text { Rathakrishna } \\
\text { et al. }{ }^{28}, 2016\end{array}$ & 1 minuto & EDTA a $17 \%$ & $5 \mathrm{ML}$ & SERINGA & NÃO & MEV & $\begin{array}{l}\text { A água super-oxidada comer- } \\
\text { cialmente disponível como } \\
\text { Oxum, provou ser igualmente } \\
\text { eficaz na remoção da smear } \\
\text { layer com menos erosão quan- } \\
\text { do comparada ao EDTA }\end{array}$ \\
\hline $\begin{array}{l}\text { Influence of Root Canal Ta- } \\
\text { pering on Smear Layer Re- } \\
\text { moval. }\end{array}$ & $\begin{array}{l}\text { Zarei et al. }{ }^{81}, \\
2016\end{array}$ & 2 minutos & EDTA a $17 \%$ & $1 \mathrm{ML}$ & SERINGA & NÃO & MEV & $\begin{array}{l}\text { Diferentes conicidades de pre- } \\
\text { paros dos canais não tiveram } \\
\text { efeito sobre a eficácia dos ir- } \\
\text { rigantes na remoção de smear } \\
\text { layer. EDTA foi mais eficaz no } \\
\text { terço médio do que apical }\end{array}$ \\
\hline $\begin{array}{l}\text { Scanning Electron Micros- } \\
\text { copic Evaluation of Smear } \\
\text { Layer Removal Using Isolated } \\
\text { or Interweaving EDTA with } \\
\text { Sodium Hypochlorite. }\end{array}$ & $\begin{array}{l}\text { da Silva Be- } \\
\text { raldo et al. }{ }^{77} \\
2017\end{array}$ & 15 segundos & EDTA a $17 \%$ & $5 \mathrm{ML}$ & SERINGA & NÃO & MEV & $\begin{array}{l}\text { A utilização de EDTA + Na- } \\
\text { OCl como irrigação final, foi } \\
\text { eficiente na remoção de sme- } \\
\text { ar layer no terço cervical e } \\
\text { médio do dente }\end{array}$ \\
\hline $\begin{array}{l}\text { Influence of passive ultrasonic } \\
\text { irrigation on the efficiency of } \\
\text { various irrigation solutions in } \\
\text { removing slemar layer: a scan- } \\
\text { ning eléctron microscope study }\end{array}$ & $\begin{array}{l}\text { Koçak et al. }{ }^{58} \text {, } \\
2017\end{array}$ & - & EDTA a $17 \%$ & $6 \mathrm{ML}$ & SERINGA & PUI & MEV & $\begin{array}{l}\text { A ativação da irrigação com } \\
\text { PUI aumentou a atividade do } \\
\text { EDTA quando comparado à } \\
\text { irrigação clássica, na remo- } \\
\text { ção da smear layer }\end{array}$ \\
\hline $\begin{array}{l}\text { Mixture of alkaline tetraso- } \\
\text { dium EDTA with sodium } \\
\text { hypochlorite promotes in vi- } \\
\text { tro smear layer removal and } \\
\text { organic matter dissolution } \\
\text { during biomechanical pre- } \\
\text { paration }\end{array}$ & $\begin{array}{l}\text { Tartari et al. }{ }^{28} \text {, } \\
2017\end{array}$ & $\begin{array}{l}5,10 \text { e } 15 \\
\text { minutos }\end{array}$ & $\begin{array}{l}\text { EDTANa }{ }_{4} \text { a } \\
10 \%, 17 \% \text { e } \\
20 \%\end{array}$ & $10 \mathrm{ML}$ & IMERSÃO & Ultrassom & MEV & $\begin{array}{l}\text { Mistura de EDTA tetrasódico alcali- } \\
\text { no }\left(\mathrm{EDTANa}_{4}\right) \text { com } \mathrm{NaOCl} \text { promo- } \\
\text { ve maior remoção de smear layer } \\
\text { in vitro e dissolução da matéria or- } \\
\text { gânica durante o PQM. No entan- } \\
\text { to, a mistura de } \mathrm{NaOCl} \text { e EDTANa } \\
\text { deve ser realizada imediatamente } \\
\text { antes de usar para evitar a redução } \\
\text { de cloro livre disponível }\end{array}$ \\
\hline $\begin{array}{l}\text { Effect of Laser-Activated Irri- } \\
\text { gations on Smear Layer Re- } \\
\text { moval from the Root Canal } \\
\text { Wall }\end{array}$ & $\begin{array}{l}\text { Wang et al. }{ }^{69} \text {, } \\
2017\end{array}$ & 1 minuto & EDTA a $17 \%$ & $5 \mathrm{ML}$ & SERINGA & LASER & MEV & $\begin{array}{l}\text { LASER + NaOCl + EDTA foi } \\
\text { o tratamento mais eficaz na } \\
\text { remoção de smear layer, po- } \\
\text { dendo ser considerado um } \\
\text { protocolo eficaz }\end{array}$ \\
\hline
\end{tabular}




\section{Discussão}

A base da terapia endodôntica está na prática efetiva dos procedimentos clínicos de esvaziamento, preparo e obturação do SCR ${ }^{31}$. Na busca de canais isentos de micro-organismos, toxinas, restos de tecidos vitais ou em decomposição, usualmente empregam-se agentes químicos e instrumentos endodônticos, os quais permitem o saneamento do sistema radicular. A limpeza e a desinfecção do SCR constituem um dos objetivos do $\mathrm{PQM}^{32,33}$

A ação mecânica dos instrumentos endodônticos sobre a parede dentinária libera raspas de dentina e resíduos orgânicos, que, misturados às substâncias químicas, formam uma substância amorfa que tende a impregnar a superfície dentinária, sedimentando-se com mais intensidade na porção apical do canal $^{34}$. McComb e Smith (1975) foram os primeiros a reportarem a presença de tal substância amorfa na parede dentinária criada pela instrumentação, a qual é chamada de smear layer.

A smear layer é constituída de dois componentes confluentes: os componentes orgânicos que são proteínas coaguladas, tecido pulpar vital ou necrótico, processos odontoblásticos, saliva, células sanguíneas e micro-organismos Os componentes inorgânicos são os minerais dos tecidos dentinários, tais como Cálcio, Fósforo, Sódio, dentre outros ${ }^{35}$.

A difusão de medicamentos intracanais como o hidróxido de cálcio no interior de túbulos dentinários aumenta quando a smear layer é removida, uma vez que ela serve de barreira física contra esses medicamentos ${ }^{36}$. Ainda nessa mesma visão, tem sido demonstrado que a remoção da smear layer promove não só a permeabilidade dentinária como também permite uma grande penetração dos cimentos obturadores no interior dos canais laterais e acessórios ${ }^{21,37-39}$. Além disso, a smear layer, acumula micro-organismos em sua composição, o que pode ser a causa de um insucesso futuro ${ }^{40}$.

Com o intuito de maximizar a remoção da lama dentinária por completo-matéria orgânica e inorgânica- o $\mathrm{NaOCl}$ dissolvendo os componentes da porção orgânica deixaria apenas as partes inorgânicas para os quelantes, que atuariam assim, de forma eficaz. Alternativa como o EDTA tem sido recomendada, pois foi verificado que essa substância, amplamente usada como um agente quelante na Endodontia, reage com os íons de cálcio da dentina removendo-os ${ }^{10}$. Além disso, estudos ${ }^{41-43}$, têm demonstrado que o EDTA realiza a descalcificação mais rápida e de forma mais eficaz, independente do $\mathrm{pH}$ e do intervalo de tempo, quando comparado com outras soluções, tais como o ácido cítrico e 0 ácido ortofosfórico.

Em se tratando da eficiência doEDTA, vários são os fatores, que irão influenciar diretamente na ação dessa substância; dentre eles, pode-se destacar o tempo de contato da solução, concentração, $\mathrm{pH}$, agitação da solução, tensão superficial, temperatura da solução e volume final de irrigante. Alguns autores ${ }^{42,45,46}$ através de suas diferentes pesquisas, concluíram que as soluções são tempo dependentes, ou seja, quanto maior o tempo de contato entre a solução e a parede dentinária, maior será a capacidade de remoção de matéria inorgânica.

Um estudo ${ }^{47}$ avaliando a limpeza do SCR após o uso do EDTA, em diferentes intervalos de tempoum, três, cinco e sete minutos- concluíram que a aplicação por sete minutos removeu quase completamente a smear layer, enquanto que com um minuto foi negligenciável a remoção.

Alguns estudos ${ }^{48,50,51}$ corroboram com o fato de um minuto não ser suficiente para remover a smear layer, especialmente no terço apical, sugerindo o uso do EDTA em um intervalo de tempo superior.

Um cuidado, no entanto, deve ser tomado quando da utilização prolongada do EDTA. Estudos comparativos analisaram os efeitos das substâncias queladoras quanto à preservação estrutural e bioquímica da matriz dentinária, ou seja, quais substâncias causam erosões peritubulares e intertubulares. O EDTA demonstrou causar tais deformações estruturais de acordo com o tempo de exposição e de concentração ${ }^{52,53}$. Com 10 minutos de exposição ele se mostrou capaz de remover a smear layer, desobstruir os túbulos dentinários, porém, passou a apresentar erosões peri e intertubulares ${ }^{52-55}$. Tais achados de erosão, foram encontrados em outro trabalho, ${ }^{25}$ que observou que a irrigação com EDTA-C a 17\%, também com um tempo prolongado de 10 minutos, não deve ser usado por provocar erosão da dentina peritubular.

Com o intuito de otimizar a utilização do EDTA, removendo a smear layer em um menor intervalo de tempo sem contudo, causar danos estruturais à dentina, métodos coadjuvantes à irrigação foram introduzidos, afim de ativar e conduzir mais apicalmente as soluções no interior do canal ${ }^{56}$. Guerisoli et al..$^{57}$ (2002) propuseram a utilização do ultrassom para agitar as soluções e verificaram que a ação dos quelantes foi efetivada quando em maior contato com as superfícies dentinárias, através do carreamento do EDTA conduzido pelo ultrassom. O mesmo foi encontrado, em um estudo comparando a eficácia de diferentes soluções irrigadoras quando da irrigação clássica com seringa e irrigação passiva ultrassônica (PUI). Os resultados demonstraram que PUI aumentou a capacidade de remoção de smear layer, para todas soluções avaliadas, inclusive o EDTA, quando comparado à irrigação clássica ${ }^{58}$.

Trabalhos ${ }^{59-61}$ registraram que tanto o EDTA, EDTA-T e o ácido cítrico foram eficazes quando usados em menor espaço de tempo, sem contudo, causar danos a estrutura dentinária. Resultados estes também encontrado por outro autor ${ }^{24}$ que reforça que para a limpeza dos canais radiculares é preciso que haja um protocolo de irrigação final e que esta irrigação se dá de forma eficiente, por três minu- 
tos com uma lavagem alternada de EDTA e $\mathrm{NaOCl}$, para a remoção da smear layer.

Frente a esses achados e aos diversos novos métodos coadjuvantes de agitação da irrigação, recomenda-se a agitação do EDTA, por um minuto, seguido da agitação do $\mathrm{NaOCl}$, para a remoção efetiva de ambos os componentes, orgânicos e inorgânicos, da camada de smear layer após o preparo biomecânico dos canais radiculares, o que permite uma adequada desinfecção do SCR, sem no entanto alterar a estrutura dentinária ${ }^{63,64}$.

Uma nova proposta de ativação do EDTA, por meio da utilização de LASER de baixa potência, também foi avaliada $27,58,65-69$. Verificou-se que o uso de LASER de diodo de baixa potência associado ao EDTA e ao $\mathrm{NaOCl}$ diminuíram a quantidade de smear layer, de todas as paredes do canal radicular. Ao comparar a tecnologia LASER com o ultrassom, ambos foram capazes de remover smear layer, no entanto, o LASER de diodo demonstrou ser um método mais eficiente para remover a camada de esfregaç ${ }^{66-68}$.

Ainda com o intuito de facilitar a penetração de soluções até a porção apical do dente, alguns autores $^{8,30}$ compararam a tensão superficial das soluções sozinhas e acrescidas de substâncias que têm a capacidade de diminuir a tensão superficial dos quelantes e concluíram que não há aumento na habilidade de remoção de Cálcio quando a tensão superficial de agentes queladores é diminuída. Por outro lado, verificaram ${ }^{70}$ que, aquelas soluções com menor tensão superficial, foram mais eficazes em remover smear layer e abrir os túbulos dentinários.

Em se tratando da variação de concentração, os autores foram unânimes ao demonstrarem que o potencial de remoção de smear layer, não se eleva com o aumento da concentração das substâncias ${ }^{30,60}$. A concentração de $17 \%$ foi relatada como sendo eficaz em $86 \%$ dos estudos.

Também foram unânimes os achados quanto ao $\mathrm{pH}$ das soluções: quanto mais ácido o $\mathrm{pH}$ (pH original) maior a capacidade das soluções em remover smear layer e desobstruir túbulos dentinários ${ }^{74,75}$. No entanto, um estudo recentemente publicado ${ }^{23}$ contradiz a literatura ao afirmar que, a mistura de EDTA tetrasódico alcalino $\left(\mathrm{EDTANa}_{4}\right)$ associado ao NaOCL promove maior remoção de smear layer, desde que a mistura de ambas as soluções seja realizada imediatadamente antes do uso, para evitar a redução de íons cloro livre disponíveis. Dessa forma, estudos futuros comparando-se os diferentes $\mathrm{pH}$ devem ser realizados.

Em se tratando da temperatura de uso do EDTA, registraram ${ }^{76}$ que a remoção de detritos foi maior quando da utilização da solução a $25^{\circ} \mathrm{C}$ e $37^{\circ} \mathrm{C}$ em comparação a $4^{\circ} \mathrm{C}$. A esse resultado, pode ser justificado o a fato de que maiores temperaturas liberam maior quantidade de íons Cloro, o que favorece a remoção da porção orgânica da smear layer realizada pelo $\mathrm{NaOCl}$, consequentemente reduzindo o seu tamanho durante a avaliação microscópica.
Vale ressaltar que a totalidade dos trabalhos produzidos, utilizaram a avaliação microscópica através de MEV, para avaliar a remoção de smear layer e desobstrução dos túbulos dentinários promovida pelos quelantes.

A literatura mostra ainda, que a variação no volume da solução irrigadora, não demonstrou ter interferência no estudo realizado por Crupton et al. ${ }^{36}$ (2005), pois $1 \mathrm{~mL}$ de EDTA aplicado por um minuto teve o mesmo efeito daquele obtido por $10 \mathrm{~mL}$ por um minuto, sugerindo assim, a utilização de $1 \mathrm{~mL}$ para que o tratamento endodôntico seja realizado com mais rapidez e menos fadiga para o operador, bem como, menor custo operacional. Apesar dessa informação, a maioria dos estudos aqui pesquisa$\operatorname{dos}^{20,24,26,28,29,30,51,56,69,76,77}$ utilizaram $5 \mathrm{~mL}$ de EDTA e encontraram resultados satisfatórios na remoção de detritos.

Quando as soluções testadas foram analisadas em diferentes segmentos do dente -terço cervical, médio, e apical- os autores verificaram que a eficiência está diretamente relacionada com: a profundidade de penetração da agulha de irrigação no interior do canal, a curvatura apical e o tamanho do diâmetro apical. Portanto, os terços, cervical e médio, apresentaram-se mais limpos, enquanto que o terço apical, demonstrou-se com maior quantidade de smear layer e menor número de túbulos desobstruídos ${ }^{30,79,80}$.

Um estudo recentemente publicado, corrobora com os achados sobre os terços cervical e médio, e ainda sugere que a irrigação durante a instrumentação do canal, seja alternada entre $5 \mathrm{~mL}$ de EDTA a $17 \%$ por 15 segundos e $5 \mathrm{~mL}$ de $\mathrm{NaOCl}$ a $2,5 \%$ por 15 segundos, para que uma maior quantidade de smear layer seja removida, principalmente no terço apical ${ }^{77}$.

Discordando em relação ao diâmetro do preparo apical dos canais, Zarei et al. ${ }^{81}$ (2016) registraram que diferentes conicidades de preparos apicais dos canais não tiveram efeito sobre a eficácia dos irrigantes na quantidade de smear layer removida.

Assim, com base nos resultados dos trabalhos avaliados nesta revisão integrativa, verificou-se que o EDTA a $17 \%$ utilizado por um período de um minuto foi ideal para a remoção da smear layer, enquanto que a aplicação de EDTA no tempo superior a sete minutos provocou erosão da dentina peritubular.

\section{Conclusão}

Pode-se concluir que uma lavagem contínua com $5 \mathrm{ml}$ de EDTA a $17 \%$, durante um minuto, seguida do uso de $\mathrm{NaOCl}$, remove de forma efetiva a camada de smear layer, sem no entanto, causar alterações inter e peritubulares. A associação entre EDTA e $\mathrm{NaOCl}$, torna tais soluções mais eficazes quando comparadas aos seus efeitos separadamen- 
te. Além disso, verificou-se que ambas as soluções quando ativadas, seja pelo método de agitação das soluções ou pela utilização de LASER, aumentaram ainda mais a remoção da smear layer.

\section{Abstract}

Objective: this study aimed to identify which EDTA application time is more often used for effectively removing the inner smear layer from root canals and which factors may influence the action of EDTA. Methodology: an integrative literature review was performed using SCIELO, Lilacs, PubMed, and Science Direct databases with the following descriptors: EDTA, final rinse, smear layer, time of irrigation, decalcification, and microhardness. Inclusion criteria were full published articles in English and Portuguese, dated from January 2000 to January 2017. Results: after analysis based on the inclusion criteria, reading of the available articles, and evaluation of the questionnaire produced, a total of 507 bibliographic references were identified in Scielo, of which three of them were selected. In Lilacs, 35 articles were identified, of which two were admitted. In PubMed, 450 references were identified, of which only one was admitted. In Science Direct, 282 articles were found, of which 24 were selected. Therefore, 30 articles were included in this review. Conclusion: based on the literature studied, it was verified that the time of EDTA use directly interfered with the quality of smear layer removal. Furthermore, it was found that using $17 \%$ EDTA for one minute was ideal for removing the smear layer, whereas EDTA application for over seven minutes caused peritubular dentin erosion.

Keywords: EDTA. Smear Layer. Chelating Agents.

\section{Referências}

1. Ingle JI, Beveridge EE, Glick BH. A terapêutica endodôntica moderna. In: Ingle JI, Beveridge EE. Endodontia. 2. ed. Rio de Janeiro: Interamericana; 1979. p. 1-55.

2. Lopes HP, Siqueira Júnior JF, Elias CN. Preparo químico-mecânico dos canais radiculares. In: Lopes HP, Siqueira Júnior JF. Endodontia: Biologia e Técnica. Rio de Janeiro: Medsi; 2004. p. 419-480.

3. Scelza MF, Pierro V, Scelza P. Effect of three different time periods of irrigation with EDTA-T, EDTA and citric acid on smear layer removal. Oral Surg. Oral Med. Oral Pathol. Oral Radiol Endod 2004; 98(4):499-503.

4. Violich DR, Chandler NP. The Smear Layer in endodontics- a review. Int Endod J 2010; 43(1):2-15.

5. Van Der Sluis LWM, Shemesh H, Wu MK, Wesselink PR. An evaluation of the influence of passive ultrasonic irrigation on the seal of root canal fillings. Int Endod J 2007; 40(5):356-361.

6. Senia ES, Marshall FJ, Rosen S. The solvent action of sodium hypochlorite on pulp tissue of extracted teeth. Oral Surg Oral Med Oral Pathol 1971; 31(1):96-103.

7. Hulsmann M, Heckendorff M, Lennon A. Chelating agents in root canal treatment: mode of action and indications for their use. Int Endod J 2003; 36(12):810-830.

8. Zehnder M. Root canal irrigants. J Endod 2006; 32(5):389398.
9. Haapasalo M, Shen Y, Qian W, Gao Y. Irrigation in endodontics. Dent Clin North Am 2010; 54(2):291-312.

10. Sen BH, Wesselink PR, Turkun M. The smear layer: a phenomenon in root canal therapy. Int Endod J 1995; 28(3):141-148.

11. Von Der Fehr FR, Nygaard-Ostby B. Effect of EDTAC and sulfuric acid on root canal dentine. Oral Surg Oral Med Oral Pathol 1963; 16(2):199-205.

12. Mccomb D, Smith DC. A preliminary scanning electron microscopic study of root canals after endodontic procedurs. J Endod 1975; 1(7):238-242.

13. Baumgartner JC, Mader CL. A scanning electron microscopic evaluation of four root canal irrigation regimens. J Endod 1987; 13(4):147-157.

14. Garberoglio R, Becce, C. Smear layer removal by root canal irrigants: a comparative scanning electron microscopic study. Oral Surg. Oral Med.Oral Pathol 1994; 78(3):359-367.

15. Çalt S, Serper A. Smear layer removal by EGTA. J Endod 2000; 26(8):459-461.

16. Ercole FF, Melo L, Alcoforado CLGC. Revisão integrativa versus revisão sistemática. Rev Min Enferm 2014; 18(1):9-11.

17. Ursi ES. Prevenção de lesões de pele no perioperatório: revisão integrativa da literatura [Dissertação de Mestrado]. Ribeirão Preto: Universidade de São Paulo; 2005.

18. Polit DF, Beck CT. Using research in evidence-based nursing practice. In: Polit DF, Beck CT.Essentials of nursing research. Methods, appraisal and utilization. Philadelphia: Lippincott Williams \& Wilkins; 2006. p. 457-494.

19. Beye ASC, Nicoll LH. Writing an integrative review. AORN J 1998; 67(4):877-880.

20. Turk T, Kaval ME, Sen BH. Evaluation of the smear layer removal and erosive capacity of EDTA, boric acid, citric acid and desy clean solutions: an in vitro study. BMC Oral Health 2015; 3(15):104.

21. Teixeira CS, Felippe M, Felippe W. The effects of aplication time of EDTA and NaOCL on intracanal smear layer removal: Na SEM analysis. Int Endod J 2005;38(5):285-290.

22. Nasher R, Franzen R, Gutknecht N. The effectiveness of the Erbium: Yttrium aluminum garnet PIPS technique in comparison to diferente chemical solutions in removing the endodontic smear layer-an in vitro profilometric study. Laser Med Sci 2016; 31(9):1871-1882.

23. Tartari T, Oda DF, Zancan RF, da Silva TL, de Moraes IG, Duarte MA, Bramante CM. Mixture of alcaline tetrasodium EDTA with sodium hypochlorite promotes in vitro smear layer removal and organic matter dissolution during biomechanical preparation. Int Endod J 2017; 50(1):106-114.

24. Mello I, Kammerer BA, Yoshimoto D, Macedo MC, Antoniazzi JH. Influence of final rinse technique on ability of Ethylenediaminetetraacetic acid of removing smear layer. J. Endod 2010; 36(3):512-514.

25. Darda S, Madria K, Jamenis R, Heda A, Khanna A, Sardar L. An in vitro evaluation of effect of EDTAC on root dentin with respect to time. J Int Oral Health 2014; 6(2):22-27.

26. Attur K, Joy MT, Karim R, Anil Kumar VJ, Deepika C, Ahmed H. Comparative analysis of endoontic smea layer removal efficacy of $17 \%$ ethylenediaminetetraacetic acid, $7 \%$ maleic acid, and $2 \%$ chlorhexidine using scanning eléctron microscope: an in vitro study. J Int Soc Prev Community Dent 2016; 6(2):160-165.

27. Ekin SNA, Erdemir A. Comparison of diferente irrigation activation techniques ons mear layer removal: an in vitro study. Microsc Res Tec 2015; 1-10. 
28. Rathakrishnan M, Sukumaran VG, Subbiya A. To evaluate the efficacy of an innovative irrigant ons mear layer removal- SEM analysis. J Clin Diagn Res 2016; 10(40:104-106.

29. Kuruvilla A, Jaganath BM, Krishnegowda SC, Ramachandra PK, Johns DA, Abraham A. A comparative evaluation of smear layer removal by using edta, etidronic acid, and maleic acid as root canal irrigants: an in vitro scanning eléctron microscopi study. J Conserv Dent 2015; 18(3): 247-251.

30. Lui JN, Kuah HG, Chen NN. Effect of EDTA with and without surfactants or ultrassonics on removal of smear layer. $\mathrm{J}$ Endod 2007; 33(4):472-475.

31. Antoniazzi JH, Silva REB. Avaliação da limpeza promovida pelo EDTA e Dakin utilizados com o último instrumento no preparo das paredes do canal radicular. Ecler endod $1999 ; 1(1)$.

32. Carvalho AS, Camargo CH, Valera MC, Camargo SE, Mancini MN. Smear layer removal by auxiliary chemical substances in biomechanical preparation: a scanning electron microscope study. J. Endod 2008; 34(11):1396-1400.

33. Kumar VR, Bahuguna N, Manan R. Comparison of efficacy of various root canal irrigation systems in removal of smear layer generated at apical third: an SEM study. J Conserv Dent 2015; 18(3):252-256

34. Gesteira MF, Silva SJA, Araúlo RPC, Lenzi H, Rocha MCBS. Ação do EDTA sobre a camada residual nos terços cervical, médio e apical do canal radicular. Rev ci méd biol. 2003; 2(2):208-218.

35. Zehnder M, Schicht O, Sener B, Scmidlin P. Reducing surface tension in endodontic chelator solutions has no effect on their ability to remove calcium from instrumented root canals. J Endod 2009; 31(8):590-592.

36. Crupton BJ, Goodell GG, Mcclanahan SB. Effects on smear layer and debris removal with varying volumes of $17 \%$ REDTA after rotary instrumentation. J. Endod 2005; 31(7):536-538.

37. Baumgartner JC, Johal S, Marshall JG. Comparison of the antimicrobial efficacy of $1.3 \% \mathrm{NaOCl}$, Biopure MTAD to $5.25 \% \mathrm{NaOCl}, 15 \%$ EDTA for root canal irrigation. J. Endod 2007; 33(1):48-51.

38. Di Lenarda R, Cadenaro M, Sbaizero O. Effectiveness of 1 mol L citric acid and 15\% EDTA irrigation on smear layer removal. Int. Endod. J. 2000; 33(1): 46-52.

39. Souza SMG, Silva TL. Demineralization effect of EDTA, EGTA, CDTA and citric acido $\mathrm{n}$ root dentin: a comparative study. Braz Oral Res 2005; 19(3):188-192.

40. Malheiros CF, Marques MM, Gavine, G. In vitro evaluation of the cytotoxic effects of acid solutions used as canal irrigants. J Endod 2005; 31(10):746-748.

41. De-Deus G, Zehnder M, Reis C, Fidel RA, Galan J Jr, Paciornik S. Longitudinal Co-site optical microscopy study on the chelating ability of etdronate and EDTA using a comparative single-tooth model. J Endod 2008; 34(1):71- 75.

42. González-López SG, Camejo-Aguilar D, Sanchez-Sanchez P, Bolaños-Carmona V. Effect of CHX on the decalcifying effect of $10 \%$ citric acid, $20 \%$ citric acid, or 17\% EDTA. J Endod 2006; 32(8):781-784.

43. Nygard-Ostby B. Chelation in root canal therapy. Etileny diamine tetracetic acid for cleasing and widening of root canals. Odont. Tdskrift 1957;65(2)3-11.

44. Saying TC. et al. Calcium loss from root canal dentin following EDTA, EGTA, EDTAC, and tetracycline- HCL treatment with or without subsequent $\mathrm{NaOCl}$ irrigation. J Endod, 2007; 33(5)581-584.
45. Yamaguchi M. Yoshida K, Suzuki R, Nakamura H. Root canal irrigation with citric acid solution. J Endod 1996; 22(1):27-29.

46. Saito K, Webb TD, Imamura GM, Goodell GG. Effect of shortened irrigation times with $17 \%$ ethylene diamine tetra-acetic acid on smear layer removal after rotary canal instrumentation. J Endod 2008; 34(8):1011-1014.

47. Sitashi P, Pan WH, Zhan L. Efficacy of solution form of ethylenediaminetetraacetic acid on removing smear layer of root canal at different exposure time in vitro. J Huazhong Univ Sci Technolog Med Sci 2014; 34(3):420-424.

48. Machado-Silveiro LF, Gozález-López S, González-Rodríguez MP. Decalcification of root canal dentine by citric acid, EDTA and sodium citrate. Int Endod J 2004;37(6):365-369.

49. Akisue E, Gavine G. Efeito das soluções de ácido cítrico a $25 \%$ e de EDTA a 17\% na dureza dentinária. Ecler 2000; 2(2).

50. Mancini M, Armellin E, Casaglia A, Cerroni L, Cianconi L. A comparative study of smear layer removal and erosion in apical intraradicular dentine with three irrigating solutions: a scanning electron microscopy evaluation. J Endod 2009; 35(6):900-903.

51. Poudyal S, Pan W, Zhang L. Efficacy of solution form of ethyleneiaminetetraacetic acid on removing smear layer of root canal at different exposure time in vitro. J Huazhong Univ Sci Technol 2014; 34(3):420-424

52. De-Deus G, Paciornik S, Mauricio MHP. Evaluation of the effect of EDTA, EDTAC and citric acido $n$ the microhardness of root dentine. Int Endod J 2009; 39(5):401-407.

53. Saying TC. Time-dependent decalcifying effects of endodontic irrigants with antibacterial properties. J Endod 2009; 35(2):280-283.

54. Çalt S, Serpes A. Time-dependent effects of EDTA on dentin structures. J Endod 2002; 28(1):17-19.

55. Semra Ç, Ahmet S. Time-depend effects of EDTA on dentin structures. J Endod 2000; 28: 17-9

56. Koçak S, Çiçek E, Saglam BC, Koçak MM, Turker SA Influence of diode laser application on the efficiency of QMiX and EDTA solutions in removing smear layer. Photomed Laser Surg 2015; 33(11):564-567.

57. Guerisoli DMZ, Marchesan MA, Walmsley AD, Lumley PJ, Pecora JD. Evaluation of smear layer removal by EDTAC and sodium hypoclorite with ultrasonic agitation. Int Endod J 2002; 35(5):418-421.

58. Koçak S, Bagci N Çiçek E, Turker SA, Saglam BC Koçak MM. Influence of passive ultrasonic irrigation on the efficiency of various irrigation solutions in removing slemar layer: a scanning electron microscope study. Microsc Res Tech 2017; 80(5):537-542.

59. Scelza MFZ, Antoniazzi JH, Scelza P. Efficacy of final irrigation-a scanning electron microscopic evaluation. J Endod $2000 ; 26(6): 355-358$

60. Scelza MFZ, Teixeira AM, Scelza P. Descalcifying effect of EDTA-T, $10 \%$ citric acid, and 17\% EDTA on root canal dentin. Oral Surg Oral Med Oral Pathol Oral Radiol En$\operatorname{dod} 2003 ; 95(2): 234-236$

61. Scelza MFZ, Pierro V, Scelza P, Pereira M. Effect of three different time periods of irrigation with EDTA-T, EDTA, and citric acid on smear layer removal. Oral Surg Oral Med Oral Pathol Oral Radiol Endod 2004; 98(4):499-503.

62. Mader CL, Baumgartner JC, Peters DD. Scanning electron microscopic investigation of the smear layer on the root canals walls. J Endod 1984; 10(10):477-483. 
63. Castro FP, Pinheiro SL, Duarte MA, Duque JA, Fernandes SL, Anchieta RB, da Silveira Bueno CE. Effect of tim and ultrasonic activation on ethylenediaminetetraacetic acido $\mathrm{n}$ smear layer removal of the root canal. Microsc Res Tech 2016; 79(11):1062-1068.

64. Çapar ID, Aydinbelge HA. Effectiveness of various irrigation activation protocols and th self-adjusting file sysstem ons mear layer and debris removal. Scanning 2014; 9999:1-8.

65. Lima GAC, Aguiar CM, Câmara AC, Alves LC, Santos FAB, Nascimento AE. Comparison of smear layer removal using the Nd: YAG Laser, ultrasond, Protaper Universal System, and Canal Brush methods: an in vivo study. J Endod 2015; 41(3):401-404.

66. Sahar- Helt S, Sarp AP, Stabholtz A, Gutkin V, Redenski I, Steinberg D. Comparison of positive-pressure, passive ultrasonic, and laser-activated irrigations ons mear-layer removal from the root canal surface. Photomed Laser Surg 2015; 33(3):129-135.

67. Amin K, Masoodi A, Nabi S, Ahmad P, Farooq R, Purra AR, Ahangar FA. Effect of diode laser and ultrasonics with and without ethlylenediaminetetraacetic acid on smear layer removal from the root canals: a scanning eléctron microscope study. J Conserv Dent 2016; 19(5):424-427.

68. Ayranci LB, Arsian H, Akcay M, Capar ID, Gok T, Saygili G. Effectiveness of laser-assisted irrigation and passive ultrassonic irrigation techniques ons mear layer removal in middle and apical thirds. Scanning 2016; 38(2):121-127.

69. Wang, X; Cheng, X., Liu, B.; Liu, X.; Yu, Q.; He, W. Effect of Laser-Activated Irrigations on Smear Layer Removal from the Root Canal Wall. Photomod laser surg 2017; 5:1-7.

70. Goldman LB, Goldman M, Kronman JH, Lin PS. The efficacy of several irrigating solutions for endodontics: a scanning electron microscopic study. Oral Surg Oral Med Oral Pathol 1981; 52(2):197-204

71. Gotze GR, Cunha CB, Primo LS, Maia LC. Effect oh the sodium hypochlorite and citric acid association on smear layer removal of primary molars. Braz Oral Res 2005; 19(4):261-266

72. Haznedaroglu, F. Efficacy of various concentrations of citric acid at different $\mathrm{pH}$ values for smear layer removal. Oral surg oral med oral pathol oral radiol endod 2003; 96(3):340344 .

73. Root canal debridement: an online study guide. J Endod 2008; 34(58):17-31.

74. Takeda FH, Harashima T, Kimura Y, Matsumoto K. A comparative study of the removal of smear layer by three endodontic irrigants and two types of laser. Int Endod J 1999; 32(1):32-39.

75. Ahir B, Parekh V, Katyanyan MK, Katyanyan PA. Smear layer removal efficacy of diferente irrigating solutions: a comparative scanning electron microscope evaluation. Indian J Dent Res 2014; 25(5):617-622.

76. Ciçek E, Keskin O. The effect of the temperature changes of EDTA and MTAD on the removal of the smear layer; a scanning electron microscopy study. Scanning 2015; 9999:1-4.

77. da Silva-Beraldo AJ, Silva RV, da Gama-Antunes AN, Silveira FF, Nunes E. Scanning electron microscopic evaluation of smear layer removal using isolated or interweaving EDTA with sodium hypochlorite. Iran Endod J 2017; 12(1):55-59.

78. Felippe MCS, Felippe WT, Espezim CS, de Freitas SF. Effectiveness of $\mathrm{NaOCl}$ alone or in combination with EDTA on the diffusion of hydroxyl ions released by calcium hydroxide paste. J Appl Oral Sci2006;14(1):1-5.
79. Khedmat S, Shokouhinejad N. Comparison of the efficacy of three chelating agents in smear layer removal. J Endod 2008; 34(5):599-602.

80. Scmidt TF, Teixeira CS, Felippe MC, Felippe WT, Pashley DH, Bortoluzzi EA. Effect of Ultrasonic Activation of Irrigants on Smear Layer Removal. J Endod 2015; 41(8):13591363.

81. Zarei M, Javidi M, Afkhami F, Tanbakuchi B, Zadeh MM, Mohammadi MM. Influence of root canal tapering on smear layer removal. N Y State Dent J 2016; 82(3):35-38.

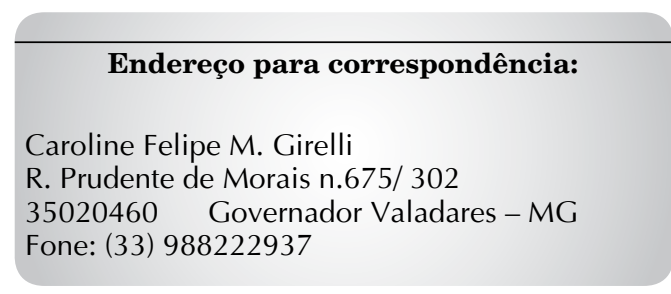

Recebido: 30/08/2016. Aceito: 05/03/2017. 